\title{
Elastic effects in the foaming of thermoplastics
}

\author{
Celeste Sagui, ${ }^{1}$ Luc Piché, ${ }^{1,2}$ Abdelhadi Sahnoune, ${ }^{2}$ and Martin Grant ${ }^{1}$ \\ ${ }^{1}$ Physics Department and Centre for the Physics of Materials, Rutherford Building, McGill University, \\ 3600 rue University, Montréal, Québec, Canada H3A 2T8 \\ ${ }^{2}$ Industrial Materials Institute, National Research Council of Canada, 75 De Mortagne Boulevard, \\ Boucherville, Québec, Canada J4B $6 Y 4$
}

(Received 17 December 1997)

\begin{abstract}
We have investigated shear and bulk elastic stresses in non-Newtonian fluids in which bubble formation due to a blowing agent is taking place. In the absence of shear, these elastic fields manifest themselves strongly during the formation of bubbles, and during coalescence events. At later times, their activity is confined to the surface of the bubbles. These effects cause a significant slow-down in radial growth. In the presence of steady shear, elasticity tends to stabilize bubble shapes, as the maximum number of droplets decreases with shear rate, while their growth rate increases. [S1063-651X(98)16109-3]
\end{abstract}

PACS number(s): $83.80 . \mathrm{Bp}, 61.25 . \mathrm{Hq}, 82.70 . \mathrm{Rr}$

Polymeric foams have gained increasing technological importance due to their light weight, low density, and high impact resistance [1]. Inside an extruder, the foams are prepared by dissolving a small molecular species, such as $\mathrm{CO}_{2}$, which acts as a blowing agent (BA) within the polymer melt. A sudden drop in pressure results in the foaming of the thermoplastic, as bubble formation and growth of the BA occurs. After nucleating from the polymer melt, the bubbles grow by diffusion of the BA, by expansion of the vapor against the melt, and by coalescence. On extrusion to a cooler temperature, the thermoplastic hardens, freezing the bubble distribution in place.

Since these foamed melts are non-Newtonian fluids, a description of bubble growth requires a set of coupled mass and momentum transfer equations augmented with the constitutive equation of the viscoelastic polymeric material. Indeed, considerable effort has been invested in such studies of bubble growth in melts [2], most of it centered on isolated bubbles surrounded by liquid. However, as mentioned above, in a foaming process a large number of bubbles grow simultaneously and interact with each other; isolated bubble models are unable to address these important aspects of the growth process. Therefore, in this paper, we take a very different approach to the traditional study of foams. We present a study of bubble growth in a polymer melt where a large number of droplets are nucleated and interact through diffusion, velocity, and particularly highly-correlated elastic fields.

We take a mesoscopic and hydrodynamic approach to polymers, which does not consider the details of molecular properties. Our model is a generalization of the two-fluid models introduced by several authors on a phenomenological basis to describe the dynamics of polymer melts and solutions [3-6]. We define a relative polymer mass concentration $\phi$; the BA concentration is given by $1-\phi$. A GinzburgLandau approximation to the Flory-Huggins free energy density gives a mixture free energy of the form

$$
F=\int d \mathbf{x}\left[f_{0} \phi^{2}(1-\phi)^{2}+\kappa(\nabla \phi)^{2}\right]
$$

where $f_{0}$ and $\kappa$ are constants, determining, for example, the surface tension. The polymer and BA velocities, $\mathbf{v}_{P}$ and $\mathbf{v}_{\mathrm{BA}}$, are related by the incompressibility condition for the average velocity $\mathbf{v}=\phi \mathbf{v}_{P}+(1-\phi) \mathbf{v}_{\text {BA }}$, i.e., $\nabla \cdot \mathbf{v}=0$. This implies that bubbles expand at the expense of the polymer. In dimensionless form, the momentum equation is

$$
\operatorname{Re} \frac{\partial \mathbf{v}}{\partial t}=\frac{\eta_{\mathrm{BA}}}{\eta^{*}} \nabla^{2} \mathbf{v}_{\mathrm{BA}}-\nabla p-\phi \nabla\left(\frac{\delta F}{\delta \phi}\right)+\nabla \cdot \sigma
$$

where $\operatorname{Re}=\rho_{0} l_{0}^{2} /\left(\eta^{*} \tau\right)$ is the Reynolds number $\left(\rho_{0}\right.$ is the melt density), $p$ is the pressure, $\eta_{\mathrm{BA}}$ is the BA viscosity, $\eta^{*}$ is the polymer melt viscosity, and $\sigma$ is the viscoelastic stress tensor. Our dimensionless results involve the characteristic length $l_{0}$ and time $\tau$.

We choose the constitutive equation for $\sigma_{i j}$ to be the linear Maxwell model of viscoelasticity. The model is $\tau \partial \sigma_{i j} / \partial t+\sigma_{i j}=\eta \dot{\gamma}_{i j}$, where $\dot{\gamma}_{i j}$ is the rate of strain [7]. (We have also considered the nonlinear upper-convected Maxwell model, but found no important differences from our results reported below.) For times long compared to $\tau$, Eq. (2) becomes the Navier-Stokes equation for a Newtonian fluid. For short times, stress is proportional to strain, via the elastic modulus, $G=\eta / \tau$. Thus an increase of elasticity in the polymer also implies an increase in its viscosity. It is convenient to separate the stress tensor into a traceless and diagonal part [8], corresponding, respectively, to shear and bulk behavior. That is, $\sigma_{i j}=\sigma_{i j}^{T}-\delta_{i j} P_{B}$, with $\operatorname{Tr}\left(\sigma^{T}\right)=0$ and $P_{B}$ $=-\operatorname{Tr}(\sigma) / d$, where $d$ is the space dimension. These quantities obey

$$
\frac{\partial \sigma_{i j}^{T}}{\partial t}+\sigma_{i j}^{T}=D \phi\left(\frac{\partial\left(\mathbf{v}_{P}\right)_{i}}{\partial x_{j}}+\frac{\partial\left(\mathbf{v}_{P}\right)_{j}}{\partial x_{i}}-\frac{2}{d} \nabla \cdot \mathbf{v}_{P}\right),
$$

and $\partial P_{B} / \partial t+P_{B}=-D \phi \nabla \cdot \mathbf{v}_{P}$, where $D=\eta / \eta^{*}, \quad \mathbf{v}_{P}=\mathbf{v}$ $+(1-\phi)\left[-\nabla(\delta F / \delta \phi)+\nabla \cdot \sigma / \phi_{0}\right] / D$ (from the incompressibility condition and the momentum equation above) $[3,5,6]$, and $\phi_{0}$ is the total (constant) concentration of the polymer. A generalization of the approach in this paper involves treating $\sigma^{T}$ and $P_{B}$ with different time constants [6]. 
Notice that since we can write $\eta=G \tau$ and $\eta^{*}=G^{*} \tau$, then $D=\eta / \eta^{*}=G / G^{*}$ is both a measure of the ratio of viscosities and of elasticity. The description of the model is completed with the diffusion equation describing the time evolution of the order parameter:

$$
\frac{\partial \phi}{\partial t}+\nabla \cdot(\phi \mathbf{v})=\frac{1}{D}\left(\phi_{0} \nabla^{2} \frac{\delta F}{\delta \phi}-\nabla \nabla: \sigma\right)
$$

We have numerically integrated Eqs. (2)-(4) in $d=2$ on a $256 \times 256$ square lattice with periodic boundary conditions, with grid size $\Delta x=1$ and time step $\Delta t=0.005$. In most foams the BA viscosity is negligible, so we set $\eta_{\mathrm{BA}}=0$. In $d=2$, the incompressibility condition is conveniently applied through a velocity potential $\Omega$, defined as $v_{x}=\partial \Omega / \partial y$ and $v_{y}=-\partial \Omega / \partial x$, where $\nabla^{2} \Omega=-\nabla \times \mathbf{v}=-\omega$, and $\omega$ is the vorticity. Thus we use the differential equation for $\Omega$ in Fourier space and, after transforming back into real space, we compute the velocity components. To ensure numerical stability when fast variations of elastic fields occur near the surface of a droplet, we use a low-pass, fast-Fourier filter [6]. Parameters used in the simulation are $\left(f_{0}, \kappa, \phi_{0}, D\right)$ $=(8,4,0.75,1-10)$. We consider an applied shear that is triangular, involving a characteristic rate of strain $V_{0}=0$ -1.2 . The shear profile is $v_{0 x}=2 V_{0} y / 256$ if $1 \leqslant y \leqslant 128$ and $v_{0 x}=2 V_{0}(1-y / 256)$ if $128<y \leqslant 256$. The constant strain rate is $\dot{\gamma}=\partial v_{0 x} / \partial y= \pm V_{0} / 128$. In Fig. 1 , we use the quantity $\dot{\gamma}_{0}=\dot{\gamma}\left(V_{0}=1\right)=1 / 128$ to normalize the stress. Using $G^{*}$ $=10^{5} \mathrm{~Pa}, \tau=0.01 \mathrm{~s}$, and $l_{0}=10^{-5} \mathrm{~m}$, our shear rate is $\dot{\gamma}$ $\leqslant 1 \mathrm{~s}^{-1}$ (corresponding to features in the total stress [6] at $\sim 3000 \mathrm{~Pa}$ ) and the surface tension is $\sim 10^{-2} \mathrm{~N} / \mathrm{m}$. For these parameters, and others where $\operatorname{Re} \leqslant 1$, the flow patterns are essentially independent of Reynolds number.

Figures 1,2, and 3 show different aspects of the effects of elastic fields on the foaming process. In the absence of applied shear, elastic fields have a large-scale effect during the initial stages of bubble formation, and decay thereafter. The fields, however, do not disappear entirely since elastic activity remains at the surface of the droplets for late times. Furthermore, long-range elastic fields are created during the coalescence of bubbles, as can be seen in Fig. 1. The left top panel shows the highly correlated structure of $\sigma_{x x}^{T}$ (shown in gray scale), which is especially prominent around two pairs of bubbles (shown in black) undergoing coalescence. The right panel shows the vectorial force field $\left(f_{i}=\partial \sigma_{i j}^{T} / \partial x_{j}\right)$, in a close-up of the top coalescing bubbles. The stress correlation function [6] also shows this behavior clearly.

The middle panels in Fig. 1 show the effect of the elastic bulk pressure $P_{B}$. Since the total volume of the mixture is kept constant, the bubbles can only expand at the expense of the polymer. Expanding bubbles, therefore, compress the polymer surrounding them, and that creates a rim of elastic pressure around the growing bubbles (shown in light gray). On the other hand, shrinking bubbles allow the surrounding polymer to decompress, and they are surrounded by a trough of elastic pressure (shown in dark gray). The barrier and trough in pressure unequivocally signal the growing and shrinking bubbles. On the right side, a later time configuration (indicated by white bubbles) is superposed on the con-

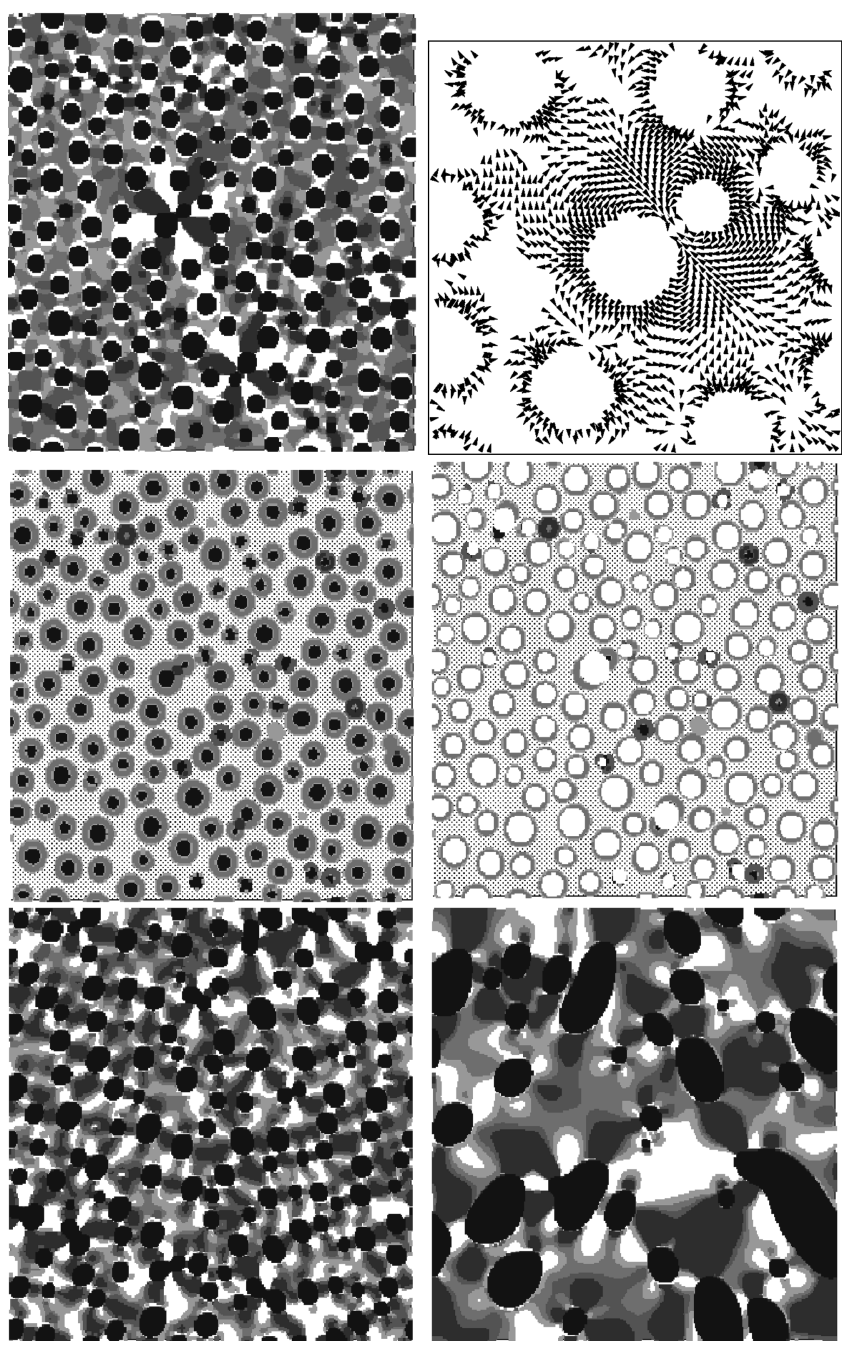

FIG. 1. Configurations for a viscoelastic system of size $256^{2}$, and viscosity ratio $D=10$. For a system without shear at $t=600$, the top panels show the stress component $\sigma_{x x}^{T}$ and the vectorial force field around a pair of coalescing bubbles. Variations in $\sigma_{x x}^{T}$ are represented by a gray scale that goes from relatively high positive values of stress for dark gray to relatively high negative values of stress for white (bubbles are represented by black). For the same system, the middle panels show the elastic bulk pressure $P_{B}$ $=-\operatorname{Tr}(\sigma) / d$. The left configuration corresponds to $t=600$; the rings of lighter gray around the bubbles correspond to positive values of $P_{B}$ (i.e., a rim of pressure) while the rings of darker gray correspond to negative values of $P_{B}$ (i.e., a trough of pressure). The right panel represents the same picture, except that a later configuration at $t=900$ (represented by white bubbles) has been superposed. Notice that the bubbles surrounded by a trough of pressure have either shrunk or disappeared. The bottom panels show the same system under shear $\left(V_{0}=0.8\right)$ at times $t=500$ and $t=1900$. The gray scale has the same meaning as in the top panel.

figuration shown on the left: we see that, in fact, the "dark gray" bubbles have disappeared or shrunk, while all the light gray bubbles remain.

Finally, the addition of shear produces strong, long-lived elastic fields as shown in the bottom panels of Fig. 1, where the stress component $\sigma_{x x}^{T}$ is shown at two times for a system undergoing shear. In the present case, where the volume remains constant and the shear viscosity does not depend on 


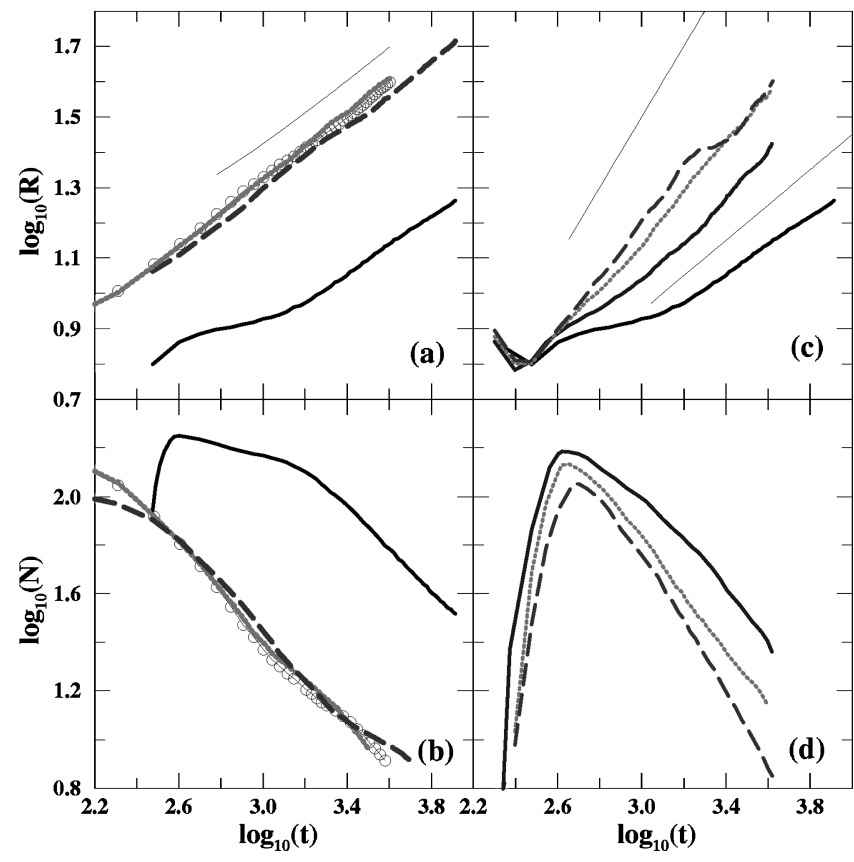

FIG. 2. Mean radius $R$ and number of droplets $N$ as function of time. The left column has no shear: solid black line, viscoelastic system with $D=10$; line with empty circles, viscoelastic system with $D=1$; long-dashed line, viscous system (no elasticity) with $D=10$; light-gray line, viscous system with $D=1$. The right column corresponds to a viscoelastic system with $D=10$ and shear rates given by $V_{0}=0.0,0.4,0.8$, and 1.2 from bottom to top in (c) and from top to bottom in (d). Thin line in (a) indicates $n=1 / 2$. Thin lines in (b) indicate $n=1$ (top) and $n=1 / 2$ (bottom).

the strain rate, these fields are nearly constant, or decay very slowly, as quantified below.

Figures 2(a) and 2(b) show the effect of viscosity and elasticity on the time variation of the mean droplet radius $R(t)$ and the total number of droplets $N(t)$ in the absence of shear. First, we compare two systems represented by Eqs. (2)-(4) with viscosity ratios $D=1$ and $D=10$. Since variations in $D$ represent variations in both viscosity and elasticity, the functions $R(t), N(t)$, and $\sigma_{x y}^{T}(t)$ [Fig. 3(a)] are quite different for these two values of $D$. To quantify the difference in terms of viscosity and elasticity, we simulate the same equations, setting the time derivatives in the Maxwell model to zero, i.e., we include viscosity and remove elasticity. For $D=1$, elasticity does not play a role in the growth of bubbles, which follows $R(t) \propto t^{n}$ with the growth exponent $n=1 / 2$, which is characteristic of off-critical quenches with hydrodynamic flows in two dimensions [9]. Increasing the viscosity (without elasticity) by an order of magnitude produces no qualitative change in the growth. However, elasticity changes this behavior completely, producing a transient plateau in $R(t)$ of very small growth, then switching to power-law growth with a very slow coarsening rate.

Figures 2(c) and 2(d) show the effect of shear for a system with $D=10$. The presence of elasticity tends to stabilize the elliptical shape of the bubble; in systems without elastic-
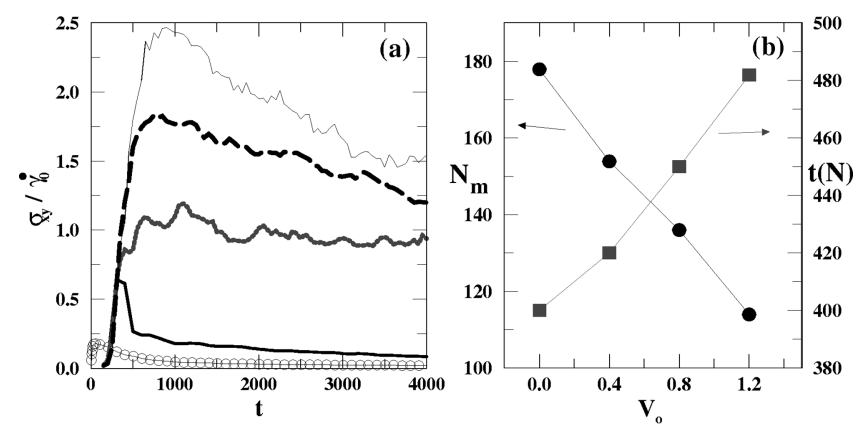

FIG. 3. (a) Normalized elastic shear stress. From bottom to top, the parameter values are (i) $V_{0}=0.0, D=1$; (ii) $V_{0}=0.0, D=10$; (iii) $V_{0}=0.4, D=10$; (iv) $V_{0}=0.8, D=10$; (v) $V_{0}=1.2, D=10$. (b) Maximum number of nucleated droplets $N_{m}$, and time $t(N)$ when that number occurs as a function of shear rate.

ity the bubbles undergo a "strip-out" instability, acquiring the shape of stripes for shear rates around $V_{0}=0.8$ and larger. For the present concentration of BA (25\%), the presence of shear rate decreases the number of nucleated droplets, and increases their rate of growth. The effective exponent is in the range $n \sim 0.7$. (We would expect $n=1$ asymptotically since a constant applied force should result in a constant velocity.) Figure 3(a) shows the time variation of the viscoelastic stress $\sigma_{x y}^{T}$ for systems with and without shear. The total shear stress $\Sigma_{x y}$ can be written as $\Sigma_{x y}$ $=\sigma_{x y}^{T}-2 \kappa \partial_{x} \phi \partial_{y} \phi+\sigma_{x y}^{0}$, where the first term is the viscoelastic contribution due to the velocity fluctuations $\mathbf{v}_{P}$ $-v_{0 x} \hat{x}$, the second term is the interface contribution, and the third term is the viscous contribution due to shear, $\sigma_{x y}^{0}$ $\simeq D \dot{\gamma}=D\left( \pm V_{0} / 128\right)$. In this figure, $\sigma_{x y}^{T}$ is normalized by $\dot{\gamma}_{0}$. Clearly, viscoelasticity, and particularly applied stress, cause dramatic qualitative increases in the internal stress. Figure 3(b) shows the maximum number of nucleated droplets $N_{m}$, and the time when that maximum number occurs $t(N)$, as a function of $V_{0}:$ As $V_{0}$ is increased, $N_{m}$ decreases sharply as $t(N)$ increases.

We expect these two-dimensional results to be observable experimentally in $d=3$, but several issues should be borne in mind. First, experiments often consider a large $\phi \sim 0.90$, compared to 0.75 considered herein. With low concentrations of BA, there will be a more marked transient regime of diffusive growth, where bubbles grow by absorbing material from the supersaturated matrix with an exponent $n \simeq 1 / 2$, while their number stays almost constant. Second, there are different growth laws for bubbles in three dimensions. In $d$ dimensions, the characteristic hydrodynamic exponent is $n$ $=1 / d$ in high-viscosity systems, when gravitational effects are negligible, while gravity speeds up growth [10]. Indeed, most experiments [1] find growth exponents that vary from $n=1 / 3$ to $n=1 / 2$ in the absence of shear.

This work was supported by the Natural Sciences and Engineering Research Council of Canada, and le Fonds pour la Formation de Chercheurs et l'Aide à la Recherche du Québec. 
[1] C. D. Han, Multiphase Flow in Polymer Processing (Academic Press, New York, 1981); MRS Bull. 21 (1996).

[2] L. E. Scriven, Chem. Eng. Sci. 10, 1 (1959); J. R. Street, A. L. Fricke, and L. P. Reiss, Ind. Eng. Chem. Fundam. 10, 197 (1971); M. Amon and C. D. Denson, Polym. Eng. Sci. 24, 1026 (1984); A. Arefmanesh and S. G. Advani, Rheol. Acta 30, 274 (1991); S. K. Goel and E. J. Beckman, AIChE. J. 41, 357 (1995); J. G. Lee and R. W. Flumbert, J. Colloid Interface Sci. 184, 335 (1996); A. Arefmanesh, S. G. Advani, and E. E. Michaelides [Polym. Eng. Sci. 30, 1330 (1990)] consider unidimensional flow with bubbles aligned along the axis of a mold cavity.

[3] F. Brochard and P. G. de Gennes, Macromolecules 10, 1157 (1977); D. Lhuiller, J. Phys. (Paris) 44, 303 (1983); E. Helfand and H. Fredrickson, Phys. Rev. Lett. 62, 2468 (1989); S.T. Milner, ibid. 66, 1477 (1991); M. Doi and A. Onuki, J. Phys. II 2, 1631 (1992); S. T. Milner, Phys. Rev. E 48, 3674 (1993).

[4] D. J. Durian, Phys. Rev. Lett. 75, 4780 (1995).
[5] T. Taniguchi and A. Onuki, Phys. Rev. Lett. 77, 4910 (1996); T. Sun, A. C. Balazs, and D. Jasnow, J. Chem. Phys. 107, 7371 (1997); H. Tanaka and T. Araki, Phys. Rev. Lett. 78, 4966 (1997); M. Criado-Sancho, J. Casas-Vazquez, and D. Jou, Phys. Rev. E 56, 1887 (1997).

[6] C. Sagui, L. Piché, and M. Grant (unpublished).

[7] R. B. Bird, R. C. Armstrong, and O. Hassager, Dynamics of Polymeric Liquids, Vol. 1: Fluid Mechanics (WileyInterscience, New York, 1987).

[8] S. P. Levitsky and Z. P. Shulman, Bubbles in Polymeric Liquids: Dynamics and Heat-Mass Transfer (Technomic, Pennsylvania, 1995).

[9] G. Leptoukh, B. Strickland, and C. Roland, Phys. Rev. Lett. 74, 3636 (1995); Y. Wu, F. I. Alexander, T. Lookman, and S. Chen, ibid. 74, 3852 (1995).

[10] F. Perrot, P. Guenoun, T. Baumberger, D. Beysens, Y. Garrabos, and B. Le Neindre, Phys. Rev. Lett. 73, 688 (1994). 